\title{
The usage of Autologous Platelet Rich Plasma in Cases of Mid-Shaft Tibial Fracture Fixed by Interlocking Nail Tibia
}

\author{
*Sherif Mohammed Abd ElMaguid Mohammed, Khaled Mohammed Emara, Mootaz Fouad Thakeb and Ahmed \\ Rayyan Ahmed
}

Orthopedic Surgery Department, Faculty of Medicine, Ain Shams University, Egyptia

Submission: August 04, 2019; Published: September 19, 2019

*Corresponding author: Sherif Mohammed Abd ElMaguid Mohammed, Orthopedic Surgery Department, Faculty of Medicine, Ain Shams University, Egypt

\begin{abstract}
Background: The tibia is one of the most commonly fractured long bones in the body. Tibial fractures are also associated with a high incidence of delayed union \& nonunion fractures (35\% to 65\% of all nonunion fractures).

Aim of the Work: to know the effect of platelet rich plasma (PRP) injection in the conditions of mid-shaft tibial fracture fixed by Inte Locking Nail Tibia (ILNT) by making a comparison between injected \& non injected cases as regard functional outcome and possible complications \& duration of healing.
\end{abstract}

Patients and Methods: 24 patients with mid-shaft tibial fracture fixed with ILNT: 12cases injected with Autologous (PRP) and 12cases without injection at Ain-Shams University Hospitals and will be arranged in randomized manner.

Conclusion: PRP usage produce bone healing in a shorter time resulting in early functional usage of the limb.

Keywords: Autologous Platelet Rich Plasma-Tibial Fracture

\section{Introduction}

The tibia is one of the most commonly fractured long bones in the body [1]. Tibial fractures are also associated with a high incidence of delayed union \& nonunion fractures (35\%to65\%of all nonunion fractures)[2]. Platelets play a prominent role as one of the first responders during the acute inflammatory phase. In response to tissue damage, platelets are activated resulting in the formation of a platelet plug and blood clot for hemostasis. The alpha granules of activated platelets contain numerous proteins that influence wound healing.

These include platelet derived growth factor, transforming growth factor, insulin-like growth factor, and Factor V, among others. In the presence of calcium, Factor $\mathrm{V}$ binds to activated factor X to produce pro-thrombin activator which converts prothrombin to thrombin. Thrombin then converts fibrinogen to fibrin which binds to platelet surface receptors. This activates another series of factors which are involved in activating factor $\mathrm{X}$ via the intrinsic pathway [3]. These proteins from platelet de-granulation are partly responsible for cellular chemotaxis, proliferation, and differentiation. This includes removal of tissue debris, angiogenesis, establishing the extracellular matrix, and regeneration of the appropriate type of tissue [4].

Platelet rich plasma (PRP) therapy, like prolotherapy, is a method of injection designed to stimulate healing. "Platelet rich plasma" is defined as "autologous blood with concentrations of platelets above baseline levels," [5] which contains at least seven growth factors [6]. Normal platelet counts in blood range between $150,000 / \mu \mathrm{l}$ and $350,000 / \mu \mathrm{l}$ and average about $200,000 / \mu \mathrm{l}$. Because the scientific proof of bone and soft tissue healing enhancement has been shown using PRP with 1,000,000 platelets $/ \mu \mathrm{l}$, this is the concentration of platelets in a $5-\mathrm{ml}$ volume of plasma which is the working definition of PRP today. Lesser concentrations cannot be relied upon to enhance wound healing, and greater concentrations have not yet been shown to further enhance wound healing [7] Enhanced healing is possible when platelet concentration is increased with PRP [8].

Because it is an autogenous preparation, PRP is inherently safe and therefore free from concerns over transmissible diseases such as HIV, hepatitis, it therefore is also well accepted 
by patients [9]. PRP therapy has low risk and few side effects. Concerns such as hyperplasia have been raised regarding the use of growth factors, however there have been no documented cases of carcinogenesis, hyperplasia, or tumor growth associated with the use of autologous PRP [10]. PRP growth factors never enter the cell or its nucleus and act through the stimulation of external cell membrane receptors of adult mesenchymal stem cells, fibroblasts, endothelial cells, osteoblasts, and epidermal cells [11]. This binding stimulates expression of a normal gene repair sequence, causing normal healing „only much faster“. Therefore, PRP has no ability to induce tumor formation [12].

\section{Patients and Methods}

It is a prospective clinical study Comparing between two Case series studies conducted on 24 patients with acute mid shaft tibial fracture fixed with inter-locking nail: 12cases with Autologous PRP injection and 12cases without Autologous PRP injection at Ain-Shams University Hospitals and will be arranged in randomized manner.

\section{Inclusion Criteria}

a. Simple, closed, acute mid shaft tibial fracture in both groups.

b. Radiographic evidence of disabling fracture.

\section{Exclusion Criteria}

a. The fracture will not be associated with any soft tissue injury or medical diseases like Acute or chronic infection and Active inflammatory or connective tissue disease (i.e., lupus, RA).

b. Active non-corrected endocrine disorder potentially associated with symptoms (i.e. hypothyroidism, diabetes).

c. Severe cardiac disease.

d. Pulmonary disease requiring medication usage.

e. Furthermore, studies also suggest that patients with systemic complications due to corticosteroid use or alcohol abuse have decreased activity of bone marrow cells.

The patients will be Evaluated Preoperatively by the Following

a. Clinical evaluation by taking an accurate history followed by general and local examination.

b. Radiological evaluation: Plain X-ray for fracture assessment (eg: site\& shape).

c. Laboratory investigations to exclude other medical diseases.

\section{Operative Technique}

\section{Patient Positioning and Preparation}

With the patient under general anesthesia, the procedure is performed. The patient is placed on the operating table in a supine position with the arms stretched out to the sides. The affected leg is prepared and draped in a sterile fashion. A c-arm fluoroscope is draped with a sterile cover in a manner that will allow access for antero-posterior and lateral views.

\section{Blood Aspiration \& Fixation}

The blood (27 $\mathrm{ml}$ of venous blood) sample was drawn into three $10 \mathrm{ml}$ vacutainer tubes containing $1 \mathrm{ml}$ of $0.106 \mathrm{M}$ sodium citrate. The samples were gently agitated to thoroughly mix the anticoagulant with the blood. The fracture will be fixed by application of interlocking nail.

\section{Centrifugation}

The blood sample was then centrifuged for 15 minutes at $3200 \mathrm{rpm}$ at $22 \mathrm{c}$ resulting in the three following layers: the inferior layer composed of erythrocytes, the intermediate layer composed of leukocytes and the superior layer made up of plasma. The buffy coat layer together with the plasma layer was collected and centrifuged for another $10 \mathrm{~min}$ at $1500 \mathrm{rpm}$ to separate the leukocytes. The plasma layer was collected, and the third centrifugation step at $3200 \mathrm{rpm}$ for $10 \mathrm{mins}$ was performed to obtain a two-part plasma: the upper part consisting of platelet poor plasma and the lower part consisting of PRP. The tubes were shaken vigorously for 30 secs to suspend platelets. The buffy coat layer, consisting of platelets, was gently aspirated into a syringe in a volume of about $3 \mathrm{ml}$ of plasma.

\section{Intra-Osseous Re-injection}

Platelet-rich plasma will be injected slowly at the fracture site. The skin perforation is closed with a circumferential suture to avoid leakage of the injection.

\section{Postoperative Management}

a. Proper antibiotic and sedation in the first few days postoperatively.

b. X-ray films will be done in two views.

c. The patient was told to begin early weight bearing \&knee \& ankle ROM exercises.

\section{Follow Up Strategy}

The patients will be followed up in the out-patient clinic at regular intervals; every month for the first six months, and then every three months. This assessment will include:

\section{Clinical and Functional Assessment}

All patients will be assessed according to the percentage of expected normal function. This is based on 4 parameters including:

I. Pain that will be assessed by Visual Analogue Scale (VAS).

II. Functional activities that will be assessed by Short Musculo-skeletal Functional Assessment (SMFA) eg;( The usage of external support or walking ability). 


\section{Orthopedics and Rheumatology Open Access Journal (OROAJ)}

\section{Radiological Assessment}

I. Fracture healing that will be assessed by Radiographic Union Score for Tibial Fractures (RUST).

II. Stress fracture and other complications.

The cases will be followed up till complete healing of the fracture or nonunion of the fracture has been occurred.

\section{Results}

i. $\quad 33$ patients with acute mid shaft tibial fracture were fixed by inter-locking nail.

ii. 9 patients were excluded because they did not meet inclusion criteria \& the rest of them was arranged in randomized manner $\&$ the follow up \& analysis was after 1 day $\& 3$ weeks $\& 3$ months (Figure 1 ).

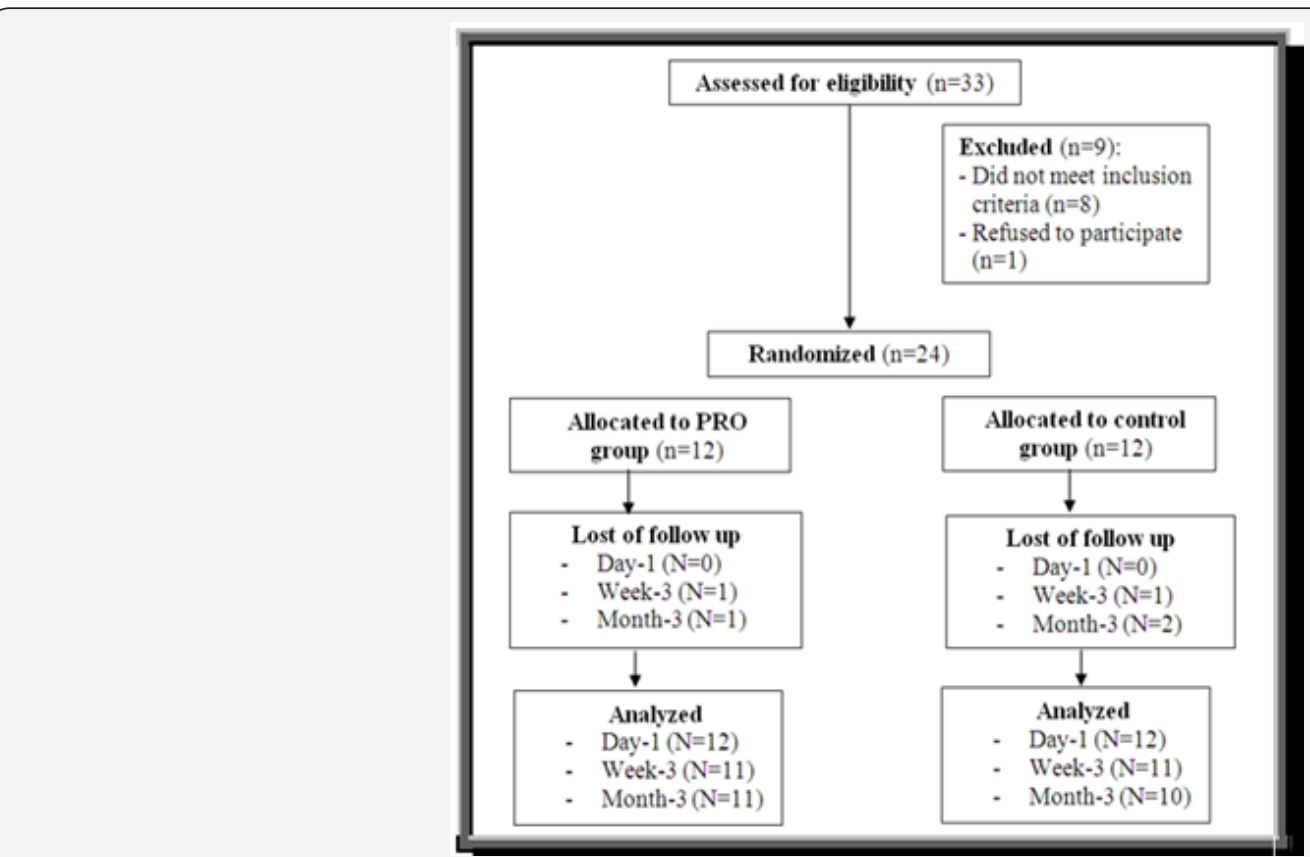

Figure 1: Patients flow chart.

iii. The patients were distributed demographically as 8(66.7\%) males\& $4(33.3 \%)$ females in PRP group \& $9(75 \%)$ males \& $3(25 \%)$ females in control group.

Table 1: Demographic characteristics among the studied groups.

\begin{tabular}{|c|c|c|c|}
\hline Items & Measure & PRP & Control \\
\hline \multirow{2}{*}{ Age(years) } & Mean \pm SD & $35.6 \pm 12.5$ & $38.5 \pm 10.7$ \\
\cline { 2 - 4 } & Range & $18.0-56.0$ & $22.0-58.0$ \\
\multirow{2}{*}{ Sex (n, \%) } & Male & $8(66.7 \%)$ & $9(75.0 \%)$ \\
\cline { 2 - 4 } & Female & $4(33.3 \%)$ & $3(25.0 \%)$ \\
\hline
\end{tabular}

^Independent t-test, \#Fisher's Exact test, SD :Standard Deviation.

b. Pain among the studied groups shows that: No significant difference between the studied groups regarding basal and day-1 Pain, then became significantly lower (better)

at week-3 and month-3 . P value at Basal (0.532), after day1 ( $0.212)$, after 3 weeks $(0.004)$, after 3 months $(<0.001)$. (Table 2).

Table 2: Demographic characteristics among the studied groups.

\begin{tabular}{|c|c|c|c|}
\hline Time & Measure & PRP & Control \\
\hline \multirow{2}{*}{ Basal } & Mean \pm SD & $70.7 \pm 5.7$ & $69.0 \pm 7.1$ \\
\cline { 2 - 4 } & Range & $61.0-79.0$ & $60.0-79.0$ \\
\multirow{2}{*}{ Day-1 } & Mean \pm SD & $64.2 \pm 5.6$ & $67.2 \pm 5.8$ \\
\cline { 2 - 4 } & Range & $55.0-72.0$ & $55.0-75.0$ \\
\hline \multirow{2}{*}{ Week-3 } & Mean \pm SD & $28.8 \pm 5.2$ & $36.5 \pm 6.0$ \\
& Range & $20.0-40.0$ & $25.0-45.0$ \\
\hline
\end{tabular}




\section{Orthopedics and Rheumatology Open Access Journal (OROAJ)}

\begin{tabular}{|c|c|c|c|c|}
\hline \multirow{2}{*}{ Month-3 } & Mean \pm SD & $4.8 \pm 3.9$ & $12.4 \pm 5.1$ & \multirow{2}{*}{$<0.001^{*}$} \\
\hline & Range & $0.0-9.0$ & $2.0-19.0$ & \\
\hline \multicolumn{5}{|c|}{ Value of PRP in pain reduction } \\
\hline \multicolumn{2}{|c|}{ Time } & \multicolumn{2}{|c|}{ Value } & $95 \% \mathrm{CI}$ \\
\hline \multicolumn{2}{|c|}{ Day-1 } & \multicolumn{2}{|c|}{$3.0 \pm 2.3$} & $-1.8-7.8$ \\
\hline \multicolumn{2}{|c|}{ Week-3 } & \multicolumn{2}{|c|}{$7.7 \pm 2.4$} & $2.7-12.7$ \\
\hline \multicolumn{2}{|c|}{ Month-3 } & \multicolumn{2}{|c|}{$7.6 \pm 2.0$} & $3.4-11.7$ \\
\hline
\end{tabular}

^Independent t-test, *Significant, Scale: 0 (Best) to 100 (worst), Cl: Confidence interval, SD :Standard Deviation.

c. Getting in or out a low chair among the studied groups became significantly lower (better) at week-3 and month-3. shows that: No significant difference between the studied groups regarding day-1 Getting in or out a low chair, then $P$ value after day1 (1.000), after 3weeks(0.022), after 3 months $(<0.001)$ ( Table 3).

Table 3: Getting in or out a low chair among the studied groups.

\begin{tabular}{|c|c|c|c|c|}
\hline Time & Measure & PRP & Control & ${ }^{\wedge} \mathbf{P}$ \\
\hline \multirow{2}{*}{ Day-1 } & Mean \pm SD & $4.8 \pm 0.5$ & $4.8 \pm 0.5$ & \multirow{2}{*}{1} \\
\hline & Range & $4.0-5.0$ & $4.0-5.0$ & \\
\hline \multirow{2}{*}{ Week-3 } & Mean \pm SD & $2.9 \pm 0.7$ & $3.6 \pm 0.7$ & \multirow{2}{*}{$0.022^{*}$} \\
\hline & Range & $2.0-4.0$ & $2.0-4.0$ & \\
\hline \multirow{2}{*}{ Month-3 } & Mean \pm SD & $1.2 \pm 0.4$ & $1.9 \pm 0.3$ & \multirow{2}{*}{$<0.001^{*}$} \\
\hline & Range & $1.0-2.0$ & $1.0-2.0$ & \\
\hline \multicolumn{5}{|c|}{ Value of PRP in getting in or out a low chair improvement } \\
\hline \multicolumn{2}{|c|}{ Time } & Value & \multicolumn{2}{|c|}{$95 \% \mathrm{CI}$} \\
\hline \multicolumn{2}{|c|}{ Day-1 } & $0.0 \pm 0.2$ & \multicolumn{2}{|c|}{$-0.4-0.4$} \\
\hline \multicolumn{2}{|c|}{ Week-3 } & $0.7 \pm 0.3$ & \multicolumn{2}{|c|}{$0.1-1.3$} \\
\hline \multicolumn{2}{|c|}{ Month-3 } & $0.7 \pm 0.2$ & \multicolumn{2}{|c|}{$0.4-1.1$} \\
\hline
\end{tabular}

^Independent t-test, *Significant, Scale: 0 (Best) to 100 (worst), Cl: Confidence interval, SD :Standard Deviation.

d. Climbing stairs among the studied groups shows lower (better) at week-3 and month-3. P value after day 1 that: No significant difference between the studied groups regarding day- 1 Climbing stairs, then became significantly (1.000) , after 3 weeks(0.030), after 3 months(0.020) (Table $4)$.

Table 4: Climbing stairs among the studied groups.

\begin{tabular}{|c|c|c|c|c|}
\hline Time & Measure & PRP & Control & ${ }^{\wedge} \mathbf{P}$ \\
\hline \multirow{2}{*}{ Day-1 } & Mean \pm SD & $4.9 \pm 0.3$ & $4.9 \pm 0.3$ & \multirow{2}{*}{1} \\
\hline & Range & $4.0-5.0$ & $4.0-5.0$ & \\
\hline \multirow{2}{*}{ Week-3 } & Mean \pm SD & $3.4 \pm 0.5$ & $3.8 \pm 0.4$ & \multirow{2}{*}{$0.030^{*}$} \\
\hline & Range & $3.0-4.0$ & $3.0-4.0$ & \\
\hline \multirow{2}{*}{ Month-3 } & Mean \pm SD & $1.1 \pm 0.3$ & $1.6 \pm 0.5$ & \multirow{2}{*}{$0.020^{*}$} \\
\hline & Range & $1.0-2.0$ & $1.0-2.0$ & \\
\hline \multicolumn{5}{|c|}{ Value of PRP in Climbing stairs improvement } \\
\hline \multicolumn{2}{|c|}{ Time } & \multicolumn{2}{|c|}{ Value } & $95 \% \mathrm{CI}$ \\
\hline \multicolumn{2}{|c|}{ Day-1 } & \multicolumn{2}{|c|}{$0.0 \pm 0.1$} & $-0.2-0.2$ \\
\hline \multicolumn{2}{|c|}{ Week-3 } & \multicolumn{2}{|c|}{$0.5 \pm 0.2$} & $0.0-0.9$ \\
\hline \multicolumn{2}{|c|}{ Month-3 } & \multicolumn{2}{|c|}{$0.5 \pm 0.2$} & $0.1-0.9$ \\
\hline
\end{tabular}

^Independent t-test, *Significant, Scale: 0 (Best) to 100 (worst), Cl: Confidence interval, SD :Standard Deviation.

e. Walking among the studied groups shows that: No significant difference between the studied groups regarding day-1 Walking then became significantly lower (better) at week-3 and month-3. P value after day1 (0.194), after 3weeks(0.039), after 3 months(0.024) ( Table 5). 


\section{Orthopedics and Rheumatology Open Access Journal (OROAJ)}

Table 5: Walking among the studied groups.

\begin{tabular}{|c|c|c|c|c|}
\hline Time & Measure & PRP & Control & ${ }^{\wedge} \mathbf{P}$ \\
\hline \multirow{2}{*}{ Day-1 } & Mean \pm SD & $4.6 \pm 0.5$ & $4.8 \pm 0.4$ & \multirow{2}{*}{0.194} \\
\hline & Range & $4.0-5.0$ & $4.0-5.0$ & \\
\hline \multirow{2}{*}{ Week-3 } & Mean \pm SD & $2.5 \pm 0.5$ & $3.0 \pm 0.6$ & \multirow{2}{*}{$0.039 *$} \\
\hline & Range & $2.0-3.0$ & $2.0-4.0$ & \\
\hline \multirow{2}{*}{ Month-3 } & Mean \pm SD & $1.2 \pm 0.4$ & $1.7 \pm 0.5$ & \multirow[t]{2}{*}{$0.024^{*}$} \\
\hline & Range & $1.0-2.0$ & $1.0-2.0$ & \\
\hline \multicolumn{5}{|c|}{ Value of PRP in Walking improvement } \\
\hline \multicolumn{2}{|c|}{ Time } & \multicolumn{2}{|c|}{ Value } & $95 \% \mathrm{CI}$ \\
\hline \multicolumn{2}{|c|}{ Day-1 } & \multicolumn{2}{|c|}{$0.3 \pm 0.2$} & $-0.1-0.6$ \\
\hline \multicolumn{2}{|c|}{ Week-3 } & \multicolumn{2}{|c|}{$0.5 \pm 0.2$} & $0.0-1.1$ \\
\hline \multicolumn{2}{|c|}{ Month-3 } & \multicolumn{2}{|c|}{$0.5 \pm 0.2$} & $0.1-0.9$ \\
\hline
\end{tabular}

^Independent t-test, *Significant, Scale: 0 (Best) to 100 (worst), Cl: Confidence interval, SD :Standard Deviation

f. Moving after sitting or lying down among the studied groups shows that: No significant difference between the Table 6: Moving after sitting or lying down among the studied groups.

\begin{tabular}{|c|c|c|c|c|}
\hline Time & Measure & PRP & Control & $\wedge_{p}$ \\
\hline \multirow{2}{*}{ Day-1 } & Mean \pm SD & $4.9 \pm 0.3$ & $4.9 \pm 0.3$ & \multirow{2}{*}{1} \\
\hline & Range & $4.0-5.0$ & $4.0-5.0$ & \\
\hline \multirow{2}{*}{ Week-3 } & Mean \pm SD & $3.3 \pm 0.5$ & $3.8 \pm 0.4$ & \multirow{2}{*}{$0.008^{*}$} \\
\hline & Range & $3.0-4.0$ & $3.0-4.0$ & \\
\hline \multirow{2}{*}{ Month-3 } & Mean \pm SD & $1.4 \pm 0.5$ & $1.9 \pm 0.6$ & \multirow{2}{*}{ 0.033* } \\
\hline & Range & $1.0-2.0$ & $1.0-3.0$ & \\
\hline \multicolumn{5}{|c|}{ Value of PRP in Moving after sitting or lying down improvement } \\
\hline \multicolumn{2}{|c|}{ Time } & \multicolumn{2}{|c|}{ Value } & $95 \%$ CI \\
\hline \multicolumn{2}{|c|}{ Day-1 } & \multicolumn{2}{|c|}{$0.0 \pm 0.1$} & $-0.2-0.2$ \\
\hline \multicolumn{2}{|c|}{ Week-3 } & \multicolumn{2}{|c|}{$0.5 \pm 0.2$} & $0.2-0.9$ \\
\hline \multicolumn{2}{|c|}{ Month-3 } & \multicolumn{2}{|c|}{$0.5 \pm 0.2$} & $0.0-1.0$ \\
\hline
\end{tabular}

^Independent t-test, *Significant, Scale: 0 (Best) to 100 (worst), Cl: Confidence interval, SD :Standard Deviation.

g. Squatting among the studied groups shows that: No significant difference between the studied groups regarding day-1 Squatting, then became significantly lower (better) studied groups regarding day-1 Moving after sitting or lying down, then became significantly lower (better) at week-3 and month-3. P value after day1 (1.000), after 3weeks(0.008), after 3 months(0.033) ( Table 6).

Table 7: Squatting among the studied groups.

\begin{tabular}{|c|c|c|c|c|}
\hline Time & Measure & PRP & Control & $\wedge \mathbf{P}$ \\
\hline \multirow{2}{*}{ Day-1 } & Mean \pm SD & $4.9 \pm 0.3$ & $4.9 \pm 0.3$ & \multirow{2}{*}{1} \\
\hline & Range & $4.0-5.0$ & $4.0-5.0$ & \\
\hline \multirow{2}{*}{ Week-3 } & Mean \pm SD & $4.2 \pm 0.4$ & $4.6 \pm 0.5$ & \multirow{2}{*}{$0.030^{*}$} \\
\hline & Range & $4.0-5.0$ & $4.0-5.0$ & \\
\hline \multirow{2}{*}{ Month-3 } & Mean \pm SD & $1.3 \pm 0.5$ & $1.8 \pm 0.4$ & \multirow{2}{*}{$0.014^{*}$} \\
\hline & Range & $1.0-2.0$ & $1.0-2.0$ & \\
\hline \multicolumn{5}{|c|}{ Value of PRP in Squatting improvement } \\
\hline & & Value & \multicolumn{2}{|c|}{$95 \% \mathrm{CI}$} \\
\hline & & $0.0 \pm 0.1$ & \multicolumn{2}{|c|}{$-0.2-0.2$} \\
\hline & & $0.5 \pm 0.2$ & \multicolumn{2}{|c|}{$0.0-0.9$} \\
\hline
\end{tabular}




\section{Orthopedics and Rheumatology Open Access Journal (OROAJ)}

Month-3

$0.5 \pm 0.2$

$0.1-0.9$

^Independent t-test, *Significant, Scale: 0 (Best) to 100 (worst), Cl: Confidence interval, SD :Standard Deviation.

h. Doing usual physical activities among the studied groups shows that: No significant difference between the studied groups regarding day-1 Doing usual physical activities, then became significantly lower (better) at week-3 and month-3. P value after day1 (1.000), after 3weeks(0.011), after 3 months(0.015) ( Table 8).

Table 8: Doing usual physical activities among the studied groups.

\begin{tabular}{|c|c|c|c|c|}
\hline Time & Measure & PRP & Control & ${ }^{\wedge} \mathbf{P}$ \\
\hline \multirow{2}{*}{ Day-1 } & Mean \pm SD & $4.9 \pm 0.3$ & $4.9 \pm 0.3$ & \multirow{2}{*}{1} \\
\hline & Range & $4.0-5.0$ & $4.0-5.0$ & \\
\hline \multirow{2}{*}{ Week-3 } & Mean \pm SD & $3.5 \pm 0.5$ & $4.1 \pm 0.5$ & \multirow{2}{*}{$0.011^{*}$} \\
\hline & Range & $3.0-4.0$ & $3.0-5.0$ & \\
\hline \multirow{2}{*}{ Month-3 } & Mean \pm SD & $1.2 \pm 0.4$ & $1.7 \pm 0.5$ & \multirow{2}{*}{$0.015^{*}$} \\
\hline & Range & $1.0-2.0$ & $1.0-2.0$ & \\
\hline \multicolumn{5}{|c|}{ Value of PRP in Doing usual physical activities improvement } \\
\hline \multicolumn{2}{|c|}{ Time } & Value & \multicolumn{2}{|c|}{$95 \% \mathrm{CI}$} \\
\hline \multicolumn{2}{|c|}{ Day-1 } & $0.0 \pm 0.1$ & \multicolumn{2}{|c|}{$-0.2-0.2$} \\
\hline \multicolumn{2}{|c|}{ Week-3 } & $0.6 \pm 0.2$ & \multicolumn{2}{|c|}{$0.2-1.1$} \\
\hline \multicolumn{2}{|c|}{ Month-3 } & $0.5 \pm 0.2$ & \multicolumn{2}{|c|}{$0.1-0.9$} \\
\hline
\end{tabular}

^Independent t-test, *Significant, Scale: 0 (Best) to 100 (worst), Cl: Confidence interval, SD :Standard Deviation.

i. Doing heavy physical activities among the studied groups shows that: No significant difference between the studied groups regarding day-1 Doing heavy physical Table 9: Doing heavy physical activities among the studied groups. activities, then became significantly lower (better) at week-3 and month-3. P value after day1 (1.000), after 3weeks(0.030), after 3 months(0.008) ( Table 9).

\begin{tabular}{|c|c|c|c|c|}
\hline Time & Measure & PRP & Control & $\mathbf{\wedge} \mathbf{P}$ \\
\hline \multirow{2}{*}{ Day-1 } & Mean \pm SD & $4.9 \pm 0.3$ & $4.9 \pm 0.3$ & \multirow{2}{*}{1} \\
\hline & Range & $4.0-5.0$ & $4.0-5.0$ & \\
\hline \multirow{2}{*}{ Week-3 } & Mean $\pm S D$ & $4.2 \pm 0.4$ & $4.6 \pm 0.5$ & \multirow{2}{*}{$0.030^{*}$} \\
\hline & Range & 4.0-5.0 & $4.0-5.0$ & \\
\hline \multirow{2}{*}{ Month-3 } & Mean \pm SD & $2.9 \pm 0.7$ & $3.7 \pm 0.5$ & \multirow{2}{*}{$0.008^{*}$} \\
\hline & Range & $2.0-4.0$ & $3.0-4.0$ & \\
\hline \multicolumn{5}{|c|}{ Value of PRP in Doing heavy physical activities improvement } \\
\hline \multicolumn{2}{|c|}{ Time } & \multicolumn{2}{|c|}{ Value } & $95 \% \mathrm{CI}$ \\
\hline \multicolumn{2}{|c|}{ Day-1 } & \multicolumn{2}{|c|}{$0.0 \pm 0.1$} & $-0.2-0.2$ \\
\hline \multicolumn{2}{|c|}{ Week-3 } & \multicolumn{2}{|c|}{$0.5 \pm 0.2$} & $0.0-0.9$ \\
\hline \multicolumn{2}{|c|}{ Month-3 } & \multicolumn{2}{|c|}{$0.8 \pm 0.3$} & $0.2-1.3$ \\
\hline
\end{tabular}

^Independent t-test, *Significant, Scale: 0 (Best) to 100 (worst), Cl: Confidence interval, SD :Standard Deviation.

j. Pivoting among the studied groups shows that: No significant difference between the studied groups regarding day-1 Pivoting, then became significantly lower (better) at week-3 and month-3. P value after day1 (0.557), after 3weeks(0.030), after 3 months(0.035) ( Table 10).

Table 10: Pivoting among the studied groups.

\begin{tabular}{|c|c|c|c|c|}
\hline Time & Measure & PRP & Control & ^P \\
\hline \multirow{2}{*}{ Day-1 } & Mean \pm SD & $4.9 \pm 0.3$ & $4.8 \pm 0.4$ & 0.557 \\
\cline { 2 - 5 } & Range & $4.0-5.0$ & $3.0-5.0$ & 0.4 \\
\multirow{2}{*}{ Week-3 } & Mean $\pm S D$ & $3.4 \pm 0.5$ & $3.0-4.0$ & $0.030^{*}$ \\
\cline { 2 - 5 } & Range & $3.0-4.0$ & $2.2 \pm 0.6$ & $0.035^{*}$ \\
\hline \multirow{2}{*}{ Month-3 } & Mean $\pm S D$ & $1.6 \pm 0.5$ & $1.0-3.0$ & \\
\cline { 2 - 5 } & Range & $1.0-2.0$ & & \\
\hline
\end{tabular}




\section{Orthopedics and Rheumatology Open Access Journal (OROAJ)}

\begin{tabular}{|c|c|c|}
\hline \multicolumn{2}{|c|}{ Value of PRP in Pivoting improvement } \\
\hline Time & Value & $95 \% \mathrm{CI}$ \\
\hline Day-1 & $-0.1 \pm 0.1$ & $-0.4-0.2$ \\
\hline Week-3 & $0.5 \pm 0.2$ & $0.0-0.9$ \\
\hline Month-3 & $0.6 \pm 0.2$ & $0.0-1.1$ \\
\hline
\end{tabular}

^Independent t-test, *Significant, Scale: 0 (Best) to 100 (worst), Cl: Confidence interval, SD :Standard Deviation.

k. Avoiding using legs among the studied groups shows that: No significant difference between the studied groups regarding day-1 Avoiding using legs, then became

Table 11: Avoiding using legs among the studied groups.

\begin{tabular}{|c|c|c|c|c|}
\hline Time & Measure & PRP & Control & $\wedge \mathbf{P}$ \\
\hline \multirow{2}{*}{ Day-1 } & Mean \pm SD & $4.7 \pm 0.5$ & $4.8 \pm 0.5$ & \multirow{2}{*}{0.67} \\
\hline & Range & $4.0-5.0$ & $4.0-5.0$ & \\
\hline \multirow{2}{*}{ Week-3 } & Mean \pm SD & $3.4 \pm 0.5$ & $3.8 \pm 0.4$ & \multirow{2}{*}{$0.030^{*}$} \\
\hline & Range & $3.0-4.0$ & $3.0-4.0$ & \\
\hline \multirow{2}{*}{ Month-3 } & Mean \pm SD & $1.1 \pm 0.3$ & $1.5 \pm 0.5$ & \multirow{2}{*}{$0.049^{*}$} \\
\hline & Range & $1.0-2.0$ & $1.0-2.0$ & \\
\hline \multicolumn{5}{|c|}{ Value of PRP in Avoiding using legs improvement } \\
\hline & & Value & \multicolumn{2}{|c|}{$95 \% \mathrm{CI}$} \\
\hline & & $0.1 \pm 0.2$ & \multicolumn{2}{|c|}{$-0.3-0.5$} \\
\hline \multicolumn{2}{|c|}{ Week-3 } & $0.5 \pm 0.2$ & \multicolumn{2}{|c|}{$0.0-0.9$} \\
\hline \multicolumn{2}{|c|}{ Month-3 } & $0.4 \pm 0.2$ & \multicolumn{2}{|c|}{$0.0-0.8$} \\
\hline
\end{tabular}

${ }^{\wedge}$ Independent t-test, *Significant, Scale: 0 (Best) to 100 (worst), Cl: Confidence interval, SD :Standard Deviation.

Table 12: Getting tired among the studied groups.

\begin{tabular}{|c|c|c|c|c|}
\hline Time & Measure & PRP & Control & ^P \\
\hline \multirow{2}{*}{ Day-1 } & Mean \pm SD & $4.5 \pm 0.5$ & $4.8 \pm 0.5$ & \multirow{2}{*}{0.223} \\
\hline & Range & $4.0-5.0$ & $4.0-5.0$ & \\
\hline \multirow{2}{*}{ Week-3 } & Mean \pm SD & $2.4 \pm 0.5$ & $2.8 \pm 0.4$ & \multirow{2}{*}{$0.030^{*}$} \\
\hline & Range & $2.0-3.0$ & $2.0-3.0$ & \\
\hline \multirow{2}{*}{ Month-3 } & Mean \pm SD & $1.2 \pm 0.4$ & $1.7 \pm 0.5$ & \multirow{2}{*}{$0.015^{*}$} \\
\hline & Range & $1.0-2.0$ & $1.0-2.0$ & \\
\hline \multicolumn{5}{|c|}{ Value of PRP in Getting tired improvement } \\
\hline \multicolumn{2}{|c|}{ Time } & \multicolumn{2}{|c|}{ Value } & $95 \% \mathrm{CI}$ \\
\hline \multicolumn{2}{|c|}{ Day-1 } & \multicolumn{2}{|c|}{$0.3 \pm 0.2$} & $-0.2-0.7$ \\
\hline \multicolumn{2}{|c|}{ Week-3 } & \multicolumn{2}{|c|}{$0.5 \pm 0.2$} & $0.0-0.9$ \\
\hline \multicolumn{2}{|c|}{ Month-3 } & \multicolumn{2}{|c|}{$0.5 \pm 0.2$} & $0.1-0.9$ \\
\hline
\end{tabular}

^Independent t-test, *Significant, Scale: 0 (Best) to 100 (worst), Cl: Confidence interval, SD :Standard Deviation.

l. Getting tired among the studied groups shows that: No significant difference between the studied groups regarding day-1 Getting tired, then became significantly lower (better) at week-3 and month-3. P value after day1 (0.223), after 3weeks(0.030), after 3 months(0.015) ( Table 12). significantly lower (better) at week-3 and month-3. P value after day1 (0.670), after 3weeks(0.030), after 3 months(0.049) ( Table 11).

ble 13: Walking with an external support among the studied groups.

\begin{tabular}{|c|c|c|c|c|}
\hline \multirow{2}{*}{ Time } & Measure & PRP & Control & ^P \\
\hline \multirow{2}{*}{ Day-1 } & Mean \pm SD & $4.6 \pm 0.5$ & $4.7 \pm 0.5$ & \multirow{2}{*}{0.689} \\
\cline { 2 - 5 } & Range & $4.0-5.0$ & $4.0-5.0$ & \\
\hline
\end{tabular}




\section{Orthopedics and Rheumatology Open Access Journal (OROAJ)}

\begin{tabular}{|c|c|c|c|c|}
\hline \multirow{2}{*}{ Week-3 } & Mean \pm SD & $2.4 \pm 0.5$ & $2.8 \pm 0.4$ & $2.0-3.0$ \\
\cline { 2 - 5 } & Range & $2.0-3.0$ & $1.5 \pm 0.5$ & $0.030^{*}$ \\
\hline \multirow{2}{*}{ Month-3 } & Mean \pm SD & $1.1 \pm 0.3$ & $1.0-2.0$ & $0.049 *$ \\
\cline { 2 - 5 } & \multicolumn{2}{|c|}{ Range } & $1.0-2.0$ & $95 \%$ CI \\
\hline \multicolumn{2}{|c|}{ Value of PRP in Walking with a limb improvement } \\
\hline \\
\cline { 2 - 5 } & Time & Value & $-0.3-0.5$ \\
\hline \\
\hline
\end{tabular}

^Independent t-test, *Significant, Scale: 0 (Best) to 100 (worst), Cl: Confidence interval, SD :Standard Deviation.

n. RUST among the studied groups shows that: No significant difference between the studied groups regarding day-1 RUST, then became significantly higher (better) at week-3 and month-3. P value after day1 (0.090) , after 3weeks(0.021), after 3 months(0.014) ( Table 14).

Table 14: RUST among the studied groups.

\begin{tabular}{|c|c|c|c|c|}
\hline Time & Measure & PRP & Control & ${ }^{\wedge} \mathbf{P}$ \\
\hline \multirow{2}{*}{ Day-1 } & Mean \pm SD & $4.5 \pm 0.5$ & $4.2 \pm 0.4$ & \multirow{2}{*}{0.090} \\
\hline & Range & $4.0-5.0$ & $4.0-5.0$ & \\
\hline \multirow{2}{*}{ Week-3 } & Mean \pm SD & $5.9 \pm 0.7$ & $5.1 \pm 0.8$ & \multirow{2}{*}{$0.021^{*}$} \\
\hline & Range & $5.0-7.0$ & $4.0-6.0$ & \\
\hline \multirow{2}{*}{ Month-3 } & Mean \pm SD & $10.5 \pm 1.0$ & $9.4 \pm 0.7$ & \multirow{2}{*}{$0.014^{*}$} \\
\hline & Range & $9.0-10.0$ & $9.0-11.0$ & \\
\hline \multicolumn{5}{|c|}{ Value of PRP in RUST improvement } \\
\hline \multicolumn{2}{|c|}{ Time } & Value & \multicolumn{2}{|r|}{$95 \% \mathrm{CI}$} \\
\hline \multicolumn{2}{|c|}{ Day-1 } & $0.3 \pm 0.2$ & \multicolumn{2}{|r|}{$-0.1-0.7$} \\
\hline \multicolumn{2}{|c|}{ Week-3 } & $0.8 \pm 0.3$ & \multicolumn{2}{|r|}{$0.1-1.5$} \\
\hline \multicolumn{2}{|c|}{ Month-3 } & $1.1 \pm 0.4$ & \multicolumn{2}{|r|}{$0.2-1.9$} \\
\hline
\end{tabular}

^Independent t-test, *Significant, Scale: 12 (Best) to 4 (worst), Cl: Confidence interval, SD :Standard Deviation.

\section{Discussion}

Today in musculoskeletal and sports medicine, PRP therapy has become highly attractive for its potential benefit and influence on repairing injured tissue, treating a wide range of degenerative disorders, and accelerating return to sport, finding its role as an injectable biologic used to augment healing of bone, tendon, ligament, muscle, and cartilage [13]. Fractures of long bones constitute most emergency operating room procedures in most trauma centers. Of these long bone injuries, tibia fractures are the most common $[14,15]$. Fractures of the tibia are brought on by a variety of high energy injury mechanisms, including motor vehicle accidents, falls, and sports injuries [16].

There are different methods of achieving skeletal stabilization. It depends on the configuration of the fracture line and the geographical location of the surgeons' practices. Intra-medullary nailing can be considered the "gold standard" for the treatment of tibial mid-shaft fractures [17]. Improved centrifugation techniques have led to the ability to concentrate platelets as PRP, with the goal of delivering these concentrates as sources of growth factors to accelerate and support the healing of hard and soft tissue injuries naturally without subjecting the patient to significant risk. One potential advantage of plateletrich preparations is that they are easily obtained from the patient's own blood after a simple centrifugation process \& can be prepared relatively in inexpensive manner.

There is a wide range of variation in the intensity and duration of centrifugation among research studies. For that reason, the lack of a suitable optimization and standardization of PRP preparation has provoked the appearance of many different platelet- rich products with controversial therapeutic effects. Studies have shown that clinical efficacy can be expected with a minimum increase in platelet concentration of 4- to 6-fold from whole blood baseline (1 million platelets/Kl) [18]. We developed a reliable and low-cost manual protocol for preparing autologous PRP, in which we achieved average a platelet concentration 4.5fold increased over baseline platelet count. PRP is prepared from autologous blood, so any concerns of allergic reactions or disease transfer are eliminated. PRP does not promote hyperplasia, carcinogenesis, or tumor growth [19].

Platelet rich plasma stimulates bone formation through its osteo-inductive effects with its rich source of growth factors [20]. Several growth factors have been shown to be expressed 
during different phases of experimental fracture healing. On the bases of these findings, it is thought that these growth factors may serve as potential therapeutic agents to enhance the repair of bone. Among these growth factors are; transforming growth factor- beta (TGF-B), bone morphogenic proteins (BMP), fibroblast growth factor (FGF), platelet-derived growth factor (PDGF), and insulin-like growth factor (IGF) [21].

The objective of the present study was to clarify whether the addition of platelet-rich plasma (PRP) would have a positive effect on bone formation in long bone fracture or not. There are few clinical studies examining the role of PRP in bone healing after orthopedic trauma [22,23]. Most studies have been related to the use of PRP in oral and cranial surgery. In trial of comparison with other studies there is no previous studies using the PRP have the same inclusion \& exclusion criteria as our research criteria as mentioned before at the beginning of the research.

About long bones, PRP has been tested in a few experimental long bone fractures models \& the clinical reports are predominantly case studies or limited case series. Most preclinical and in vivo studies agree that the application of PRP is effective in stimulating and enhancing the healing processes of the bone defects. Nevertheless, there is no complete consensus on the beneficial effect of PRP on bone fractures, delay unions and non-unions, and some researchers believe that it cannot be effective in enhancing new bone formation $[24,25]$.

Several clinical studies have applied PRP in patients with different conditions of bone defects. A total of 27 human clinical studies performed between 2012 and 2015, regarding the effects of PRP on bone healing and regeneration. Most of these studies (96\%) have used PRP with an autologous origin in which 22 studies $(81 \%)$ yielded excellent outcomes. The results obtained from two studies showed that PRP in combination with other material and scaffolds led to non-significant improvement in bone regeneration and the treatment in one study had no positive effect on bone healing after 12-week follow-up. Some clinical studies used allogenous form of PRP and to the knowledge of the authors, no study have still used the xenogenous PRP for treatment of bone defects [26].

For instance, Celio-Mariano et al. [27] estimated the effect of allogeneic PRP on alveolar bone defects in 25 patients for 6 months. The results obtained from this study confirmed the promising efficacy of the allogeneic PRP. In a study conducted by Ozdemir \& Okte [28] the efficacy of PRP in the treatment of delayed union of long bones was assessed in 132 participants with long bone fracture between 2009 and 2012. After PRP administration, bone union was established in 108 patients (81.8\%) and 24 patients (18.2\%) showed no improvement and resulted in nonunion, respectively.

The highest efficacy was related to delayed union of the proximal tibia, whereas the lowest one was associated to that of the proximal humerus and therefore, they concluded that the PRP efficacy depends on the fracture location. In addition, they showed the effect of the material used for fracture fixation on PRP efficacy, so that open reduction and plate fixation led to a much more incidence of delayed union than closed reduction with intramedullary nail fixation. Although, most studies have reported the effectiveness of PRP in enhancing and improving bone healing, the beneficial effects of PRP in clinical applications remains controversial and doubtful because of the limitations in the methodologies, follow up and study design [26]

The clinical and experimental data in the literature regarding the osteogenic potential of PRP are controversial. Several authors report a positive influence of PRP on bone regeneration [29-30]. However, our results confirm several clinical [31-32] and experimental [33] studies demonstrating the positive effect of PRP on bone healing.

Regarding pain assessment by VAS, it was found that there was significant reduction in VAS scores in PRP group than in control group(at 3 months $<0.001$ in P value \& $3.4-11.7$ in $95 \%$ CI) . Also, the other assessment methods like RUST(at 3 months $<0.014$ in P value \& $0.2-1.9$ in 95\% CI) \& SMFA(eg; Doing heavy physical activities at 3 months $<0.008$ in $\mathrm{P}$ value \& $0.2-1.3$ in $95 \% \mathrm{CI}$ ) was significantly better than the control group.

The overall results in our study revealed that there is significant better difference with PRP injection in comparison with the control group as regards healing time, pain relief \& return to normal function. A full detailed history was taken from all patient included in this study. Including date and mode of trauma, weather it was closed or open fracture, type and time of initial management also if there was another management done and its result, history of infection, history of previous similar condition, also history of systemic diseases and special habits. Clinical examination was done for all patients by inspecting Skin condition, local signs of infection, and obvious deformity of limb, palpating fracture site to assess tenderness, and growth movements if present. Antero-posterior and lateral plain x-rays views were done for all patients at time of injection.

\section{Conclusion}

a. Healing and regeneration of the bone fractures is a challenging issue in orthopedic researches.

b. PRP is considered as an ideal and cost-effective source of growth factors and bioactive compounds.

c. PRP promotes bone repair and regeneration by various mechanisms which is mainly due to its inflammatory cytokines and growth factors.

d. Based on the basic to clinical evidences, because there are several controversies regarding efficacy of PRP, it is still too soon to judge and offer such therapeutic option as an appropriate modality for promoting bone repair and regeneration. 


\section{References}

1. Heckman JD, Sarasohn Kahn J (1997) The economics of treating tibial fractures. The cost of delayed unions. Bull Hosp Joint Dis 56(1): 63-72.

2. Dickson K, Katzman S, Delgado E, Contreras D (1994) Delayed unions and non-unions of open tibial fractures. Correlation with arteriography results. Clin Orthop Relat Res (302): 189-193.

3. Pietrzak WS, Eppley BL (2005) Platelet rich plasma: biology and new technology. J Craniofacial Surgery 16(6): 1043 -1054.

4. Marx RE (2001) Platelet rich plasma (PRP): What is PRP and what is not PRP? Implant Dent 10: 225-228.

5. Hall M, Bank P, Meislin R, Jazrawi L, Cardone D (2009) Platelet-rich plasma: Current concepts and application in sports medicine. Journal of the American Academy of Orthopedic Surgeons 27: 602-608.

6. Marx R, Kevy S, Jacobson M (2008) Platelet rich plasma (PRP): A primer. Pract Pain Manag Mar 8(2): 46-47.

7. Robert E, Marx DDS (2001) Platelet-Rich Plasma (PRP): What Is PRP and What Is Not PRP? Reprinted from IMPLANT DENTISTRY. Lippincott Williams \& Wilkins Printed in U.S.A 10: 4.

8. Marx R, Garg A (2005) Dental and Craniofascial Applications of Platelet-Rich Plasma. Quintessence Publishing Co.

9. Robert E Marx, DDS (2004) Platelet-Rich Plasma: Evidence to Support Its Use. J Oral Maxillofac Surg 62: 489-496.

10. Creaney L, Hamilton B (2008) Growth factor delivery methods in the management of sports injuries: the state of play. British J Sports Med 42: 314-320.

11. Marx R (2001) The biology of platelet-rich plasma (reply to letter to the editor). J Oral Maxillofac Surg 59: 1120.

12. Schmitz JP, Hollinger J (2001) The biology of platelet-rich plasma (letter to the editor). J Oral Maxillofac Surg 59: 1119.

13. Nguyen RT, Borg Stein J, McInnis K (2011) Applications of platelet-rich plasma in musculoskeletal and sports medicine: an evidence-based approach. PMR 3(3): 226-50.

14. Court Brown CM, McBirnie J (1995) The epidemiology of tibial fractures. J Bone Joint Surg Br 77(3): 417- 21.

15. Lang GJ, Cohen BE, Bosse MJ, Kellam JF (1995) Proximal third tibial shaft fractures. Should they be nailed? Clin Orthop Relat Res 315 64-74.

16. Vidyadhara S, Sharath KR (2006) Prospective study of the clinicradiological outcome of interlocked nailing in proximal third tibial shaft fractures. Injury 37(6): 536-42.

17. Zelle BA, Bhandari M, Espiritu M, Koval KJ, Zlowodzki M (2006) Treatment of distal tibia fractures without articular involvement: a systematic review of 1125 fractures. J Orthop Trauma 20(1): 76-9.

18. Weibrich G, Hansen T, Kleis W (2004) Effect of platelet concentration in platelet-rich plasma on peri-implant bone regeneration. Bone 34: $665 Y 71$.

19. Sampson S, Gerhardt M, Mandelbaum B (2008) Platelet rich plasma injection grafts for musculoskeletal injuries: a review. Curr Rev Musculoskelet Med 1: 165 Y74.
20. Gandhi A, Doumas C, O'Connor JP (2006) Evaluations of histomorphometrical property of platelet-rich plasma on tibial bone defect healing in rabbit. Bone 38: 540-546.

21. Klein MB, Naveen Yalamanchi, Hung Pham, Michael T. Longaker, James Chan (2002) Flexor tendon healing in vitro: effects of TGFbeta on tendon cell collagen production. J Hand Surg Am 27(4): 615-20.

22. Eppley BL, Pietrzak WS, Blanton M (2006) Platelet-rich plasma: a review of biology and applications in plastic surgery. Plast Reconstr Surg 118: 147-59.

23. Fennis JP, Stoelinga PJ, Jansen JA (2004) Mandibular reconstructiion: a histological and histomorphometric study on the use of autogenous scaffolds, particulate corticocancellous bone grafts and platelet rich plasma in goats. Int J Oral Maxillofac Surg 33: 48-55.

24. Aghaloo TL, Moy PK, Freymiller EG (2002) Investigation of plateletrich plasma in rabbit cranial defects: a pilot study. J Oral Maxillofac Surg 60: 1176-81.

25. Wei LC, Lei GH, Sheng PY (2012) Efficacy of platelet-rich plasma combined with allograft bone in the management of displaced intraarticular calcaneal fractures: a prospective cohort study. J Orthop Res 30: $1570-1576$

26. Ahmad O, Soodeh A, Ali M (2015) Platelet-rich plasma for bone healing and regeneration. Expert Opinion on Biological Therapy 16(2):213-32.

27. Celio Mariano R, de Melo WM, Carneiro-Avelino C (2012) Comparative radiographic evaluation of alveolar bone healing associated with autologous platelet-rich plasma after impacted mandibular third molar surgery. J Oral Maxillofac Surg 70:19-24.

28. Ozdemir B, Okte E (2012) Treatment of intrabony defects with betatricalciumphosphate alone \& in combination with PRP. J Biomed Mater Res B Appl Biomater 100: 976-83.

29. Anitua E (1999) Plasma rich in growth factors: preliminary results of use in the preparation of future sites for implants. Int J Oral Maxillofac Implants 14: 529- 535.

30. Thorwarth M, Rupprecht S, Falk S, Felszeghy E, Wiltfang J, et al. (2005) Expression of bone matrix proteins during de novo bone formation using a bovine collagen and platelet-rich plasma (prp)-an immunohistochemical analysis. Biomaterials 26: 2575-84.

31. Froum SJ, Wallace SS, Tarnow DP, Cho SC (2002) Effect of platelet-rich plasma on bone growth and osseo- integration in human maxillary sinus grafts: three bilateral case reports. Int J Periodont Restor Dent 22: $45-53$.

32. Raghoebar GM, Schortinghuis J, Liem RS, Ruben JL, van der Wal JE, et al. (2005) Does platelet rich plasma promote remodeling of autologous bone grafts used for augmentation of the maxillary sinus floor? Clin Oral Implants Res 16: 349-56.

33. Fuerst G, Reinhard G, Tangl S, Mittlbock M, Sanroman F, et al. (2004) Effect of platelet-released growth factors and collagen type I on osseous regeneration of mandibular defects: a pilot study in minipigs.

J Clin Periodontol 31: 784-90 

(C) Commons Attribution 4.0 License

BY DOI: 10.19080/OROAJ.2019.14.555900

\section{Your next submission with Juniper Publishers will reach you the below assets}

- Quality Editorial service

- Swift Peer Review

- Reprints availability

- E-prints Service

- Manuscript Podcast for convenient understanding

- Global attainment for your research

- Manuscript accessibility in different formats ( Pdf, E-pub, Full Text, Audio)

- Unceasing customer service

Track the below URL for one-step submission https://juniperpublishers.com/online-submission.php 